\title{
Pflanzen und Menschen in Südwestchina
}

Im Sommer 2007 hatten unsere Besucherinnen und Besucher die Gelegenheit, sich an einem Tag auf die Reise in die Länder Ägypten und China zu begeben, denen jeweils eine Ausstellung gewidmet war. Die große Informationsausstellung „Pflanzen und Menschen in Südwestchina" wurde vom 19. Juli bis 21. Oktober 2007 in der Galerie Ost am Palmenhaus auf einer Fläche von rund $600 \mathrm{~m}^{2}$ gezeigt.

Die Ausstellung ist in einer Kooperation des Palmengartens mit deutschen Organisationen
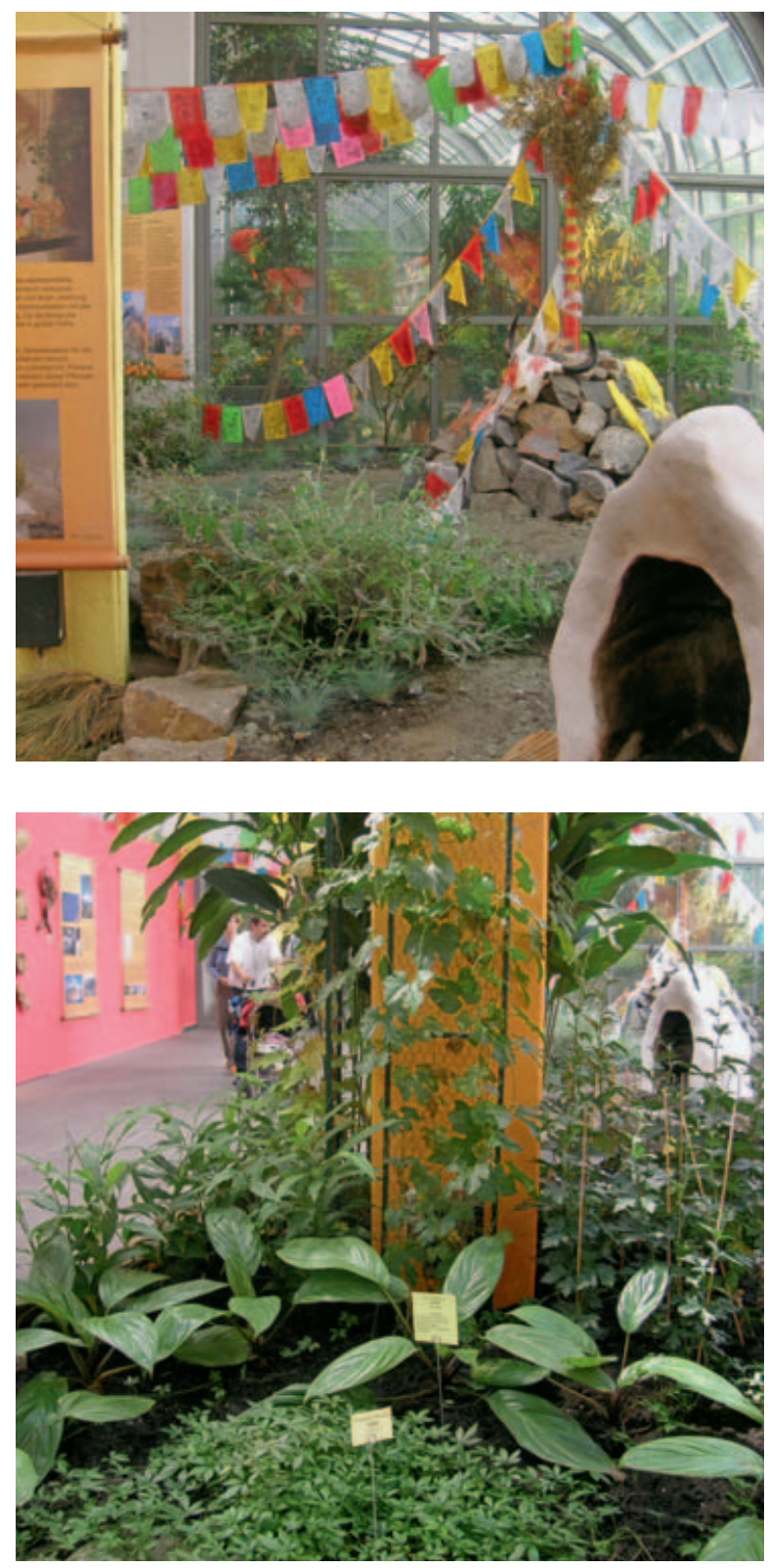

der Entwicklungszusammenarbeit (Centrum für Internationale Migration und Entwicklung, CIM, Deutsche Gesellschaft für Technische Zusammenarbeit, GTZ) und chinesischen Instituten (Centre for Biodiversity and Indigenous Knowledge, CBIK, Kunming Institute of Botany) entstanden.

Der Südwesten Chinas, besonders die Provinz Yunnan an den Ausläufern des Himalaya, hat viele Gesichter. Neben zahlreichen Volksgruppen mit eigenen Sprachen und Traditionen verfügt die Region über eine außergewöhnlich vielfältige Pflanzenwelt. Minderheiten-Kulturen und botanische Vielfalt sind durch äußere Einflüsse zunehmend gefährdet und drohen verloren zu gehen. Die Ausstellung bot einen Einblick in das traditionelle Wissen, die Verwendung von Pflanzen als Rohstoff, Nahrungsmittel oder Medizin. Sylvia Reinhardt, die die Ausstellung konzipiert hat, konnte in China vor Ort recherchieren, sodass wir hier Spannendes aus erster Hand vermitteln konnten. Zusammen mit KAI-UwE KLINKER und weiteren Helfern brachte sie $800 \mathrm{~kg}$ an Ausstellungs- und Demonstrationsmaterial nach Frankfurt.

Ein besonderer Reiz der Palmengarten-Ausstellungen besteht darin, dass Informationstafeln in einer ansprechenden Kombination von Objekten und Pflanzen gezeigt werden. Kurz hinter dem Eingang wurde ein Mani-Steinhügel mit Gebetsfahnen aus dem kargen Hochland gezeigt, dazu die entsprechenden Ritualund Räucherpflanzen erklärt. Daneben befand sich eine Apotheke mit pflanzlichen Mitteln aus der traditionellen chinesischen Medizin. Von den Nutzpflanzen wurde besonders das Thema Tee vertieft. Papier-Schöpfrahmen und verschiedene Papiersorten illustrierten das Kapitel „Traditionelle Papierherstellung in China“. Besonders grün und mit vielen Gewächsen ausge-

Abb. 1 (oben): Mani-Steinhaufen, Gebetsfahnen und Räucherkräuter.

Abb. 2 (unten): Heilpflanzen aus China. 

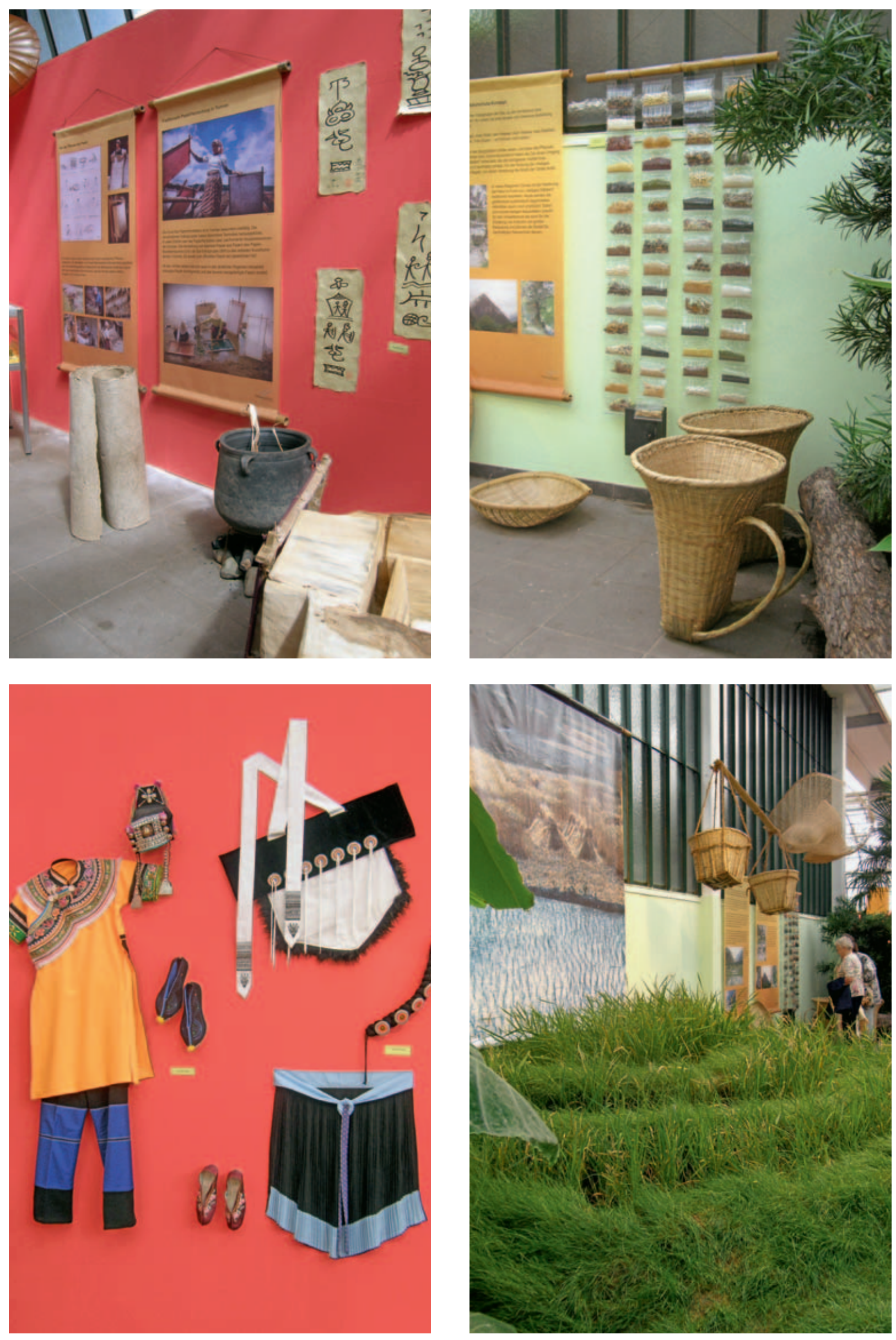

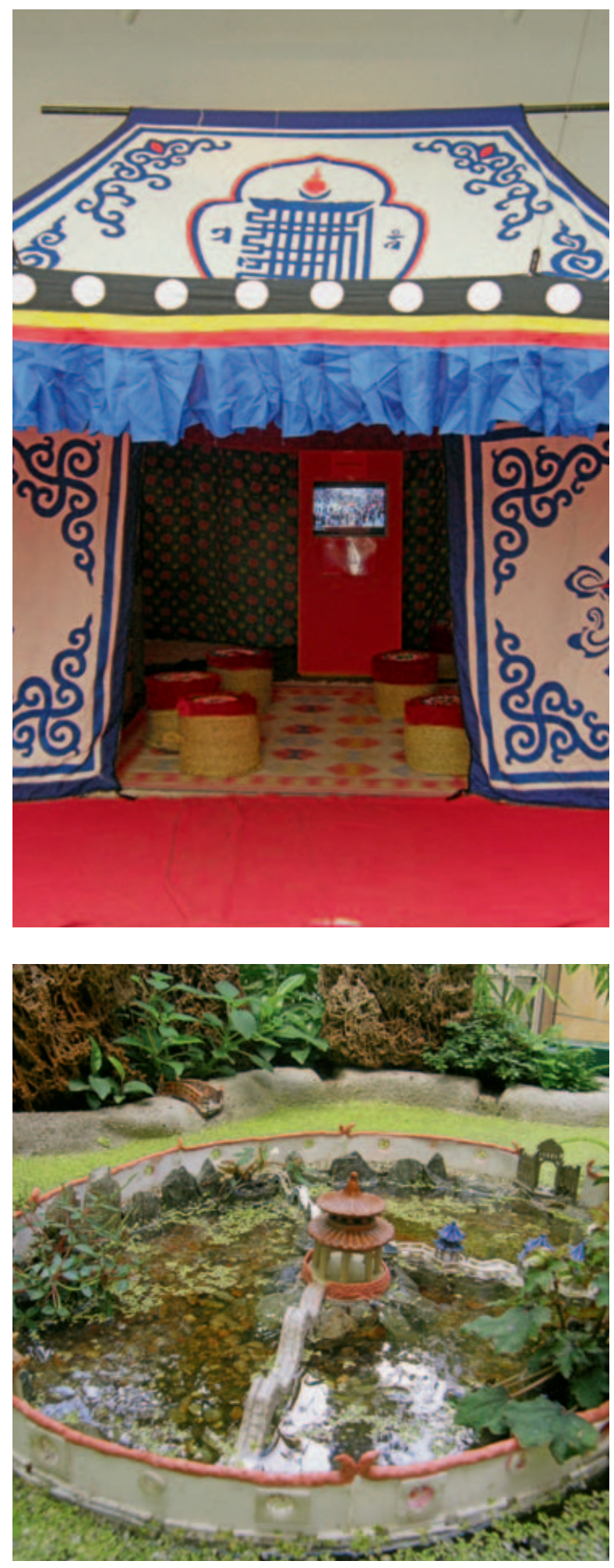

stattet war der Bereich der chinesischen (Wild)Gemüsepflanzen. Gegenüber erhob sich ein terrassiertes Reisfeld, dekoriert mit diversen Gerätschaften, die für Anbau und Ernte von Reis erforderlich sind. Ein traditioneller Landwirtschaftskalender rundete das Bild ab. Ein origi- nales tibetisches Zelt im Lesesaal bot die Möglichkeit, sich in seinem Innern einen Videofilm über traditionelle Tänze anzusehen. Darüber hinaus informierte eine Fotoausstellung der GTZ über agrarbiologische Vielfalt in China (Hainan und Hunan).

Im Eingangsbereich der Galerie gab ein chinesischer Miniaturgarten, konzipiert von MARIanne Beuchert, der auch diese Ausstellung gewidmet war, einen Einblick in die asiatische Gartenkunst. Die Beete vor dem nördlichen Eingang zur Ausstellungsgalerie wurden von der Firma Garten-Hesse gestaltet, die sich u.a. auf asiatische Gartenanlagen spezialisiert hat. Sowohl im Freiland als auch im Tropicarium war ein Rundgang zu insgesamt 40 Pflanzen ausgewiesen. Hier gab es Kurzinformationen zu bekannteren und weniger bekannten Zierpflanzen, die ursprünglich vor allem aus Südwestchina stammen. Der Rundgang endete im Tropicarium. Dort wurde im Übergangsbereich zwischen den feuchten und den trockenen Tropen eine Fotoausstellung von Holger PerNER zum Pflanzenparadies Huanglong gezeigt. Chinesische Pflanzen waren auch auf einer Führung durch den benachbarten Botanischen Garten zu erkunden.

Während des Ausstellungszeitraumes gab es verschiedene Aktionen und zahlreiche Führungen, darunter auch zu Spezialthemen wie traditionelle Papierherstellung, Tee oder wilde Gemüsespezialitäten. Die Inhalte der Ausstellung sind im Palmengarten-Sonderheft Nr. 41 vertieft.

Die Ausstellung ist als Wanderausstellung konzipiert und kann vom Palmengarten ausgeliehen werden.

Abb. 3 (S. 155, links oben): Traditionelle Papierherstellung. Abb. 4 (S. 155, rechts oben): Gewürze, verschiedene Nahrungsmittel, Ackerfrüchte und Reissorten.

Abb. 5 (S. 155, links unten): Traditionelle Kleidung ethnischer Minoritäten in Yunnan.

Abb. 6 (S. 155, rechts unten): Palmengarten-Reisfeld.

Abb. 7 (oben): Tibetisches Zelt mit Videovorführung.

Abb. 8 (unten): Chinesischer Miniaturgarten. 